\title{
Experiencias en la Aplicación de un Ciclo de Mejora en el Aula en la Asignatura de "Termodinámica"
}

\section{Some Experiences in an Improvement Cycle in Classroom to teach "Thermodynamics"}

José MARÍA DELGAdo SÁNCHEZ

Universidad de Sevilla

Dpto. de Ingeniería Energética

jdelgado17@us.es

Fecha de recepción: 29-05-2019.

Fecha de aceptación: 04-06-2019.

DOI: http://dx.doi.org/10.12795/9788447221912.006 Pр.: $153-176$ 


\section{Resumen}

En este artículo se comparten las experiencias de aplicar un Ciclo de Mejora en el Aula ciclo(CIMA) durante la asignatura de Termodinámica en el Grado de Ingeniería de las Tecnologías Industriales de la Universidad de Sevilla. El objetivo ha sido invertir la metodología aplicada para centrar la atención en los alumnos, generando situaciones que les involucren en la clase. Se trata así de evitar la "clase magistral" y que los alumnos consigan un mayor aprendizaje y a largo plazo (Beard y Hartley, 1984). Se ha planteado un nuevo modelo metodológico aplicando un aprendizaje basado en problemas.

Palabras clave: Experimentación docente universitaria, Docencia universitaria, Aprendizaje Basado en Problemas (ABP), Termodinámica, Grado en Ingeniería de las Tecnologías Industriales

\section{Abstract}

A session for the improvement in teaching has been achieved to implement new methodologies in the Thermodinamics subject, which is included in the Bachelor Degree for Industrial Engineering at the University of Seville. The main objective of this project has consisted in flipping the traditional "master class" to transfer the main role of the classroom to the students. It is expected that this alternative teaching methodology will let students get a better understanding of the concepts in the long-term (Beard and Hartley, 1984). More precisely, the proposed model is focussed on the learning procedure based on problems.

Keywords: university teaching innovation, university teaching, learning based on problems, thermodynamics, Industrial Engineering Bachelor

Jornadas de Formación e Innovación Docente del Profesorado | № 2 (2019) Esta obra se distribuye con la licencia Creative Commons 


\section{Contexto}

La puesta en práctica del Ciclo de Mejora en el Aula (CIMA) que se describe en el presente artículo se ha llevado a cabo en la asignatura de Termodinámica, de segundo curso del Grado de Ingeniería de las Tecnologías Industriales (GITI), en la Universidad de Sevilla. La asignatura consta de cuatro grupos donde la docencia se imparte por dos profesores, en sesiones de 110 minutos ininterrumpidos, con periodicidad de dos sesiones por semana cada grupo. El examen es común para todos los alumnos, lo que exige una buena coordinación entre los diferentes profesores en cuanto a contenidos, avances, etc. Tradicionalmente la asignatura presenta cierta complejidad para los estudiantes y dificultad para comprender los contenidos y superar el examen.

El Ciclo de Mejora se ha puesto en práctica durante los meses de Marzo-Abril del curso 2018/19, y en dos grupos en paralelo. Cada grupo de clase tiene aproximadamente 120 alumnos que asisten, lo que en algunas ocasiones resulte complicado implementar ciertas metodologías docentes innovadoras.

El modelo didáctico tradicional en esta asignatura está basado en la "clase magistral". El objeto por tanto de la práctica de este Ciclo de Mejora consiste en implementar alternativas innovadoras docentes, siguiendo la guía de un Aprendizaje Basado en Problemas (ABP), donde se dé una mayor participación y relevancia al alumno a cambio de desplazar al profesor a un papel secundario que actúe más como un guía en la búsqueda de conocimientos. Se buscará así, romper el binomio "Aprendizaje vs Enseñanza" clásico. Se parte de la hipótesis de que un alumno no aprende si no es consciente de la acción. Despertar la conciencia debería ser por tanto la prioridad del profesor, puesto que una vez se ha conseguido un alumno motivado y receptivo, el desarrollo de conocimientos es una tarea 
que puede hacer el alumno por si solo a base de resolver casos con complejidad creciente. La verdadera consciencia del aprendizaje es la clave del conocimiento adquirido. "El aprendizaje más profundo sucede más a menudo cuando se acompaña de la reflexión y experimentación" (Hutchens, 2009).

\section{Diseño previo del ciclo de mejora en el aula}

El modelo metodológico aplicado hasta antes de esta experiencia, replicaba el método impartido por otros profesores de la asignatura en cursos académicos anteriores. Consistía en una clase magistral dividida en dos partes: exposición de contenidos teóricos y resolución en la pizarra por parte del profesor de problemas (Figura 1). Los inconvenientes de este modelo, son: el alumno tiene un papel pasivo en la clase, no participa, no activa su curiosidad y se limita a almacenar conceptos que estudian antes del examen. Pierden la aplicabilidad de los contenidos, lo cual es fundamental para un estudiante de una carrera técnica de ingeniería.
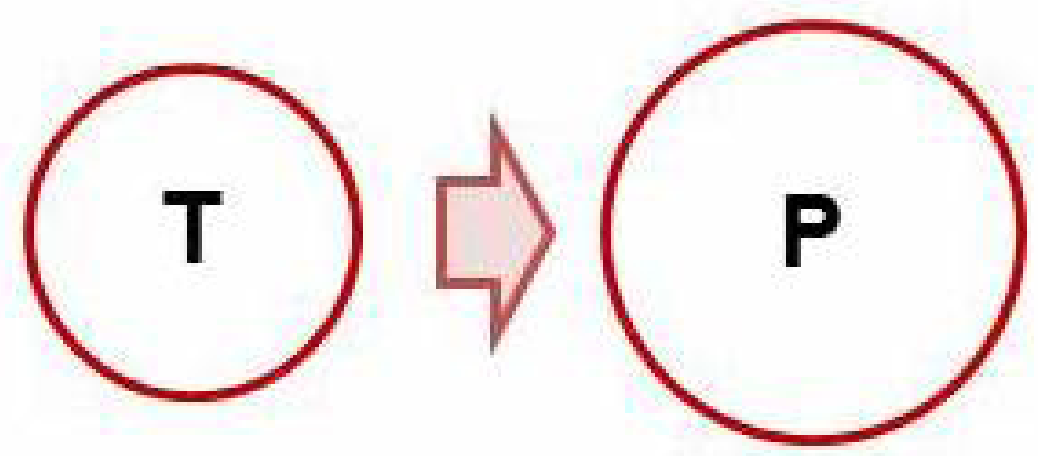

Figura 1. Modelo metodológico previo al ciclo de mejora en el aula

Para evitar estos inconvenientes, se ha propuesto un ciclo de mejora en el aula que se basa en los siguientes pilares: 
- Papel del alumno en la clase: el enfoque será que el alumno sea el centro de la clase, tanto en protagonismo como en generador de conocimientos que comparte con sus compañeros (Finkel, 2008). Se busca con esta aproximación un mayor interés, curiosidad y entusiasmo por aprender, además de fomentar competencias importantes para su desarrollo profesional, como por ejemplo la argumentación, el debate, exposición de ideas, trabajo en equipo y respeto por otras opiniones.

- Reorganización de los contenidos de la asignatura: si bien los contenidos no pueden ser modificados durante la práctica del Ciclo de Mejora, dada la coordinación con el resto de profesores/grupos, sí se propone una reorganización de los mismos para establecer una secuencia de los que ofrezca al alumno una serie de preguntas encadenadas, donde la respuesta de un problema sea la pregunta del siguiente. Para esta actividad, es crucial el diseño de mapas de contenidos que ofrezcan relaciones entre ellos.

- La metodología que se ha puesto en práctica difiere del método tradicional (Figura 1): el profesor inicia la clase con una serie de preguntas que deben atraer la atención del alumno y situarlo en el contexto del tema a abordar. El alumno es quien debe ir averiguando la solución al problema, usando los conocimientos previos y aquellos que se imparten por primera vez durante la clase. El profesor se convierte así en un mero facilitador de información, que el alumno recibe, convierte, asocia y transforma.

- Evaluación de conocimientos: se han puesto en práctica dos herramientas. En primer lugar se propone a los estudiantes de manera semanal y voluntaria (no tiene recompensa en la calificación de la asignatura) la resolución de un ejercicio, que entregarán al profesor en la semana próxima. El profesor los corrige y a su vez devuelve a los estudiantes. El beneficio es doble: al alumno se enfrenta al papel en blanco de resolver un 
problema, adelanta el momento del examen, y cuando recibe el ejercicio corregido (no calificado) por el profesor, reflexiona en sus aciertos y errores para mejorar. Al profesor le ayuda a conocer si los contenidos que se transfieren a los alumnos son comprendidos, y donde es necesario tal vez un refuerzo. Por otro lado, como segunda herramienta, se han realizado dos ejercicios de evaluación antes y después de la sesión donde se pone en práctica el Ciclo de Mejora. Permitirá al profesor construir las escaleras de aprendizaje e identificar los obstáculos que permanecen en la comprensión de los estudiantes.

Así, con estas hipótesis de partida, se plantea un nuevo modelo metodológico (Figura 2), que persigue adaptar el modelo anterior (Figura 1) a una clase participativa centrada en los alumnos.

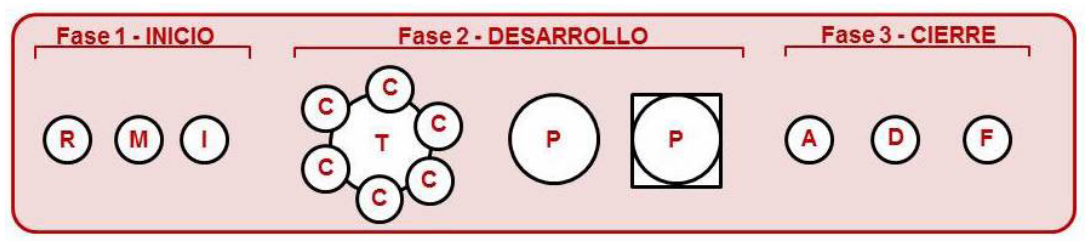

Figura 2. Nuevo modelo metodológico implementado en el Ciclo de Mejora

Las sesiones se dividen en tres fases: Fase 1 "inicio", donde el profesor repasa $(R)$ contenidos de la sesión anterior, motiva (M) a los estudiantes con el planteamiento de un problema de interés tecnológico/social relacionado con la asignatura, y termina con la exposición (I) de los objetivos y estructura de la sesión; Fase 2 "desarrollo", en la que el profesor continuamente motiva a los estudiantes con preguntas y respuestas ( $\mathrm{T}$ ) (C) para generar los Conocimientos por ellos mismos en lugar de transferirlos expositivamente por él; continua con una resolución de problema tipo en la pizarra para mostrar contenidos procedimentales (P) y a continuación son los propios estudiantes quienes trabajan entre ellos en la resolución de 
casos similares pero de complejidad avanzada de manera tutorizada por el profesor $(P)$; Fase 3 "cierre", por último el profesor propone un ejercicio voluntario (A) para trabajar fuera de clase, resuelve dudas (D) y resume las ideas principales de la sesión (F) usando el mapa de contenidos. El modelo se cierra con la actividad de evaluación de contenidos inicial y final de la sesión, que será mediante cuestionario proporcionado a los estudiantes.

Es relevante exponer la "pregunta inicial" que se les plantea a los alumnos, porque ésta actúa de tracción a su interés durante la sesión. Los alumnos en sesiones anteriores han abordado conceptos más teóricos de la disciplina como son los Principios Termodinámicos, conceptos de Energía-Calor y sus transformaciones, Exergía y trabajo útil de un sistema... pero el objetivo al que se debe enfrentar un futuro ingeniero en su desarrollo profesional en este campo es a diseñar u optimizar un proceso energético. Así, se les plantea a los estudiantes “¿Cuál es el fin de diseñar un proceso energético?", ¿Por qué la Sociedad necesita que ingenieros diseñen y mejoren procesos energéticos?"... y en un contexto medioambiental como el que vivimos, se les añade la pregunta de “¿Cómo deben ser dichos procesos energéticos respecto al medioambiente?".

En sí, el objetivo de la sesión que coincide con la implementación del Ciclo de Mejora es precisamente que los alumnos realicen un análisis termodinámico de ciclos, evalúen sus rendimientos e identifiquen puntos de mejora en el diseño. El tema en cuestión no ofrece contenidos nuevos al estudiante, sino que es la oportunidad para que implementen y pongan en práctica aquellos conceptos teóricos termodinámicos que han aprendido en las sesiones anteriores. Así, es una oportunidad ideal para que sean ellos mismos quienes sean los protagonistas del aprendizaje en la clase: tienen todas las herramientas previas, y el profesor es responsable de plantearles inquietudes y guiarles en el camino de buscar la solución. 
El Mapa de Contenidos confeccionado para esta sesión (Figura 3) ha sido útil para identificar las ideas principales, establecer relaciones entre los distintos contenidos, y generar un hilo conductor que explique cómo estos contenidos son necesarios para abordar las preguntas iniciales que se plantean a los alumnos.

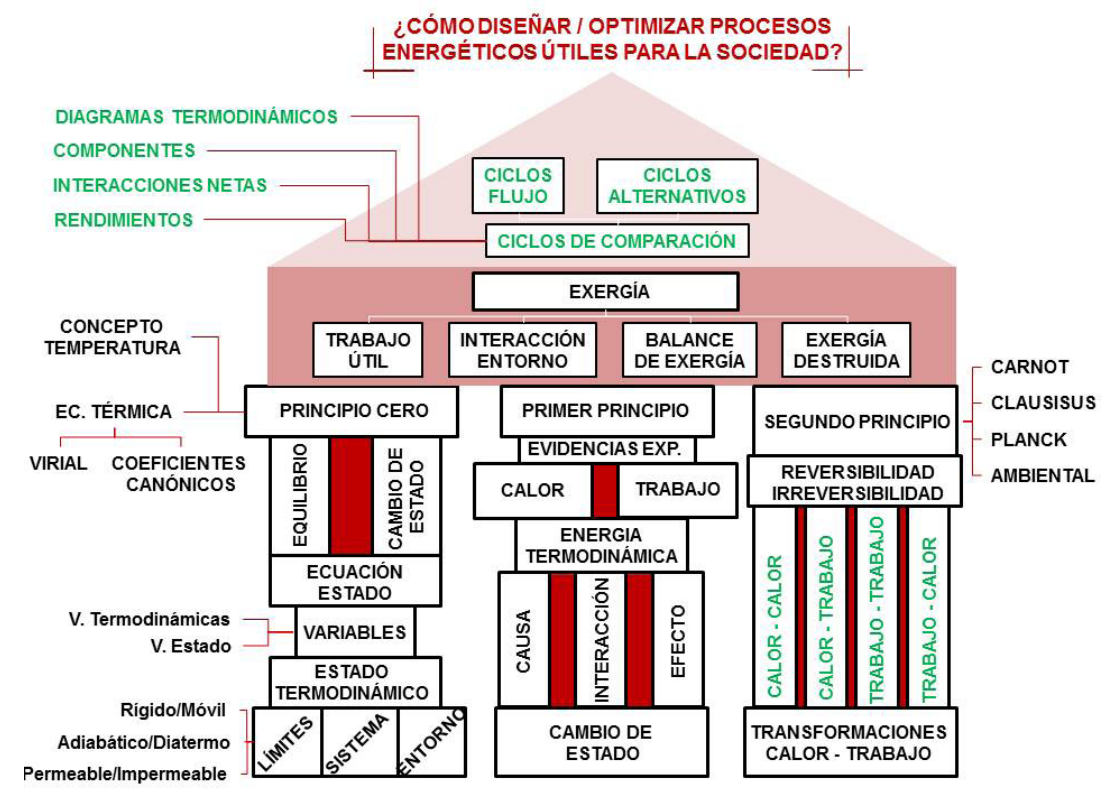

Figura 3. Mapa de Contenidos

El diseño del Mapa de Contenidos se aprovecha para transmitir a los alumnos que todos los conceptos que han aprendido en sesiones previas, son necesarias y de interés para un estudiante de ingeniería que puede desarrollar su futuro profesional en el ámbito energético. Se plantea el Mapa de Contenidos como una casa soportada por tres pilares fundamentales (matemáticamente se demuestra que tres puntos no alineados es el número mínimo de grados de libertad que definen un plano), siendo cada uno de ellos los tres Principios de la Termodinámica que han estudiado previamente. Además, se observa gráficamente 
como cada pilar está construido con conceptos alineados verticalmente que coinciden con la secuencia temporal en cómo se han explicado en clase: los conceptos se construyen unos sobre otros y ninguno queda aislado. Comprenden como cada Principio de la Termodinámica por sí mismo no aporta técnicamente a la solución, pero la combinación de los tres les permite construir un plano de conocimiento superior donde estudiar las interacciones del sistema con el entorno, realizar balances de exergía y determinar así la exergía destruida o el trabajo útil. Este nuevo plano horizontal es la base de los procesos energéticos. Sobre él podemos disponer en diferentes configuraciones componentes que han analizado individualmente y comprender como funcionan de forma colaborativa para un beneficio global. Conocidos pues los componentes que intervienen en el proceso, las interacciones energéticas que tienen lugar entre ellos, los Principios Termodinámicos que se usan en el análisis y el cálculo de los rendimientos energéticos y exergéticos, es posible diseñar o identificar puntos de mejora en cualquier proceso o ciclo termodinámico.

El planteamiento que se ha seguido en el Ciclo de Mejora es diseñar este Mapa de Contenidos inicialmente por parte del Profesor, para identificar aquellas ideas principales y las relaciones entre los distintos conceptos; pero no se muestra al alumno hasta el final de la sesión, donde se hace un Cierre (Fase 3) en el que los propios alumnos son quienes van resaltando los puntos de interés de la sesión que ven críticos, y junto a este análisis, el profesor va presentando poco a poco el Mapa de Contenidos. El resultado es positivo en cuanto las ideas principales que los alumnos proponen y la temporización con la que surgen, coincide en buena parte con el Mapa de Contenidos previo que se había realizado.

Así, el Mapa de Contenidos proporciona tres fortalezas al Ciclo de Mejora: (1) revisión inicial de los contenidos por parte del profesor; (2) soporte en el cierre de la sesión 
para identificar ideas principales aprendidas; (3) soporte que se transfiere a los alumnos como material docente de apoyo en su estudio de la asignatura.

\section{Cuestionario inicial y final: evaluación del aprendizaje}

Antes y después de la sesión del CIMA se invitó a los alumnos a cumplimentar un breve cuestionario con objeto de poder cuantificar el grado de aprendizaje de los mismos. Para ello, se diseñó un cuestionario inicial y otro final (Figura 4), que aunque tienen diferentes cuestiones abordan retos similares. Los resultados obtenidos se analizarán posteriormente mediante la creación de las escaleras de aprendizaje e identificación de obstáculos en el aprendizaje de los estudiantes.

Sin duda, esta actividad tiene que usar como entrada las salidas del Mapa de Contenidos: en base a éste se deben dirigir las preguntas que se plantean en el cuestionario (Porlán, 2017).

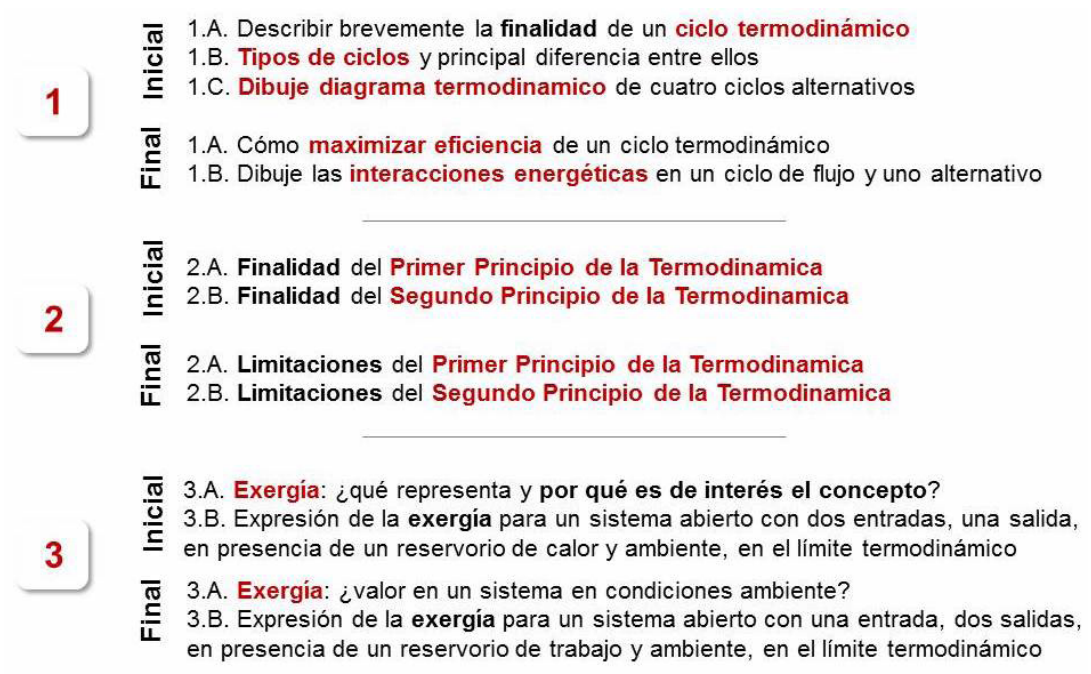

Figura 4. Cuestionario inicial y final. Evaluación del aprendizaje

Jornadas de Formación e Innovación Docente del Profesorado | № 2 (2019) Esta obra se distribuye con la licencia Creative Commons Reconocimiento-NoComercial-SinObraDerivada Internacional (CC BY-NC-ND 4.0.) 


\section{Aplicación del Ciclo de Mejora en el Aula}

A continuación (Tabla 1) se presenta un resumen de las actividades planteadas en el Ciclo de Mejora de acuerdo al Modelo Metodológico de la Figura 2. Cada actividad descrita está acompañada de una breve descripción así como los tiempos planificados y los objetivos que persigue.

Tabla 1. Secuencia de actividades del Ciclo de Mejora

\begin{tabular}{|c|c|c|c|}
\hline & Actividades & Descripción & Finalidad \\
\hline 1 & $\begin{array}{l}\text { Repaso del día } \\
\text { anterior ( } 5 \text { min) }\end{array}$ & $\begin{array}{l}\text { El profesor repasa las } \\
\text { conclusiones prin- } \\
\text { cipales de la sesión } \\
\text { anterior mediante pre- } \\
\text { guntas a los alumnos, } \\
\text { para conectar con los } \\
\text { contenidos de la se- } \\
\text { sión actual }\end{array}$ & $\begin{array}{l}\text { - Situar al alumno en } \\
\text { el aula } \\
\text { - Que el alumno se } \\
\text { concentre }\end{array}$ \\
\hline 2 & $\begin{array}{l}\text { Motivación } \\
(5 \mathrm{~min})\end{array}$ & $\begin{array}{l}\text { El profesor plantea a } \\
\text { los alumnos unas pre- } \\
\text { guntas que capten su } \\
\text { atención y sean trac- } \\
\text { toras de su curiosidad }\end{array}$ & $\begin{array}{l}\text { - Captar la atención del } \\
\text { alumno } \\
\text { - Motivar al alumno } \\
\text { - Situar al alumno } \\
\text { en los contenidos a } \\
\text { impartir }\end{array}$ \\
\hline 3 & $\begin{array}{l}\text { Introducción } \\
\text { (5 min) }\end{array}$ & $\begin{array}{l}\text { El profesor expone la } \\
\text { estructura de los con- } \\
\text { tenidos de la sesión }\end{array}$ & $\begin{array}{l}\text { - Orientar al alumno } \\
\text { en los contenidos }\end{array}$ \\
\hline 4 & $\begin{array}{l}\text { Cuestionario Ini- } \\
\text { cial (10 min) }\end{array}$ & $\begin{array}{l}\text { El profesor facilita a } \\
\text { los alumnos el cues- } \\
\text { tionario inicial }\end{array}$ & $\begin{array}{l}\text { - Evaluar conocimien- } \\
\text { tos previos }\end{array}$ \\
\hline 5 & $\begin{array}{l}\text { Teoría con } \\
\text { reflexiones } \\
(40 \text { min) }\end{array}$ & $\begin{array}{l}\text { El profesor imparte } \\
\text { contenidos teóricos } \\
\text { explicando conceptos } \\
\text { a través de preguntas } \\
\text { a los estudiantes }\end{array}$ & $\begin{array}{l}\text { - Transferencia de co- } \\
\text { nocimiento a los } \\
\text { estudiantes } \\
\text { - Facilitar herramien- } \\
\text { tas teóricas para } \\
\text { que puedan resolver } \\
\text { problemas } \\
\text { - Conocer el grado de } \\
\text { comprensión de los } \\
\text { estudiantes }\end{array}$ \\
\hline
\end{tabular}

Jornadas de Formación e Innovación Docente del Profesorado | № 2 (2019) Esta obra se distribuye con la licencia Creative Commons Reconocimiento-NoComercial-SinObraDerivada Internacional (CC BY-NC-ND 4.0.) 


\begin{tabular}{|c|l|l|l|}
\hline 6 & $\begin{array}{l}\text { Resolución Pro- } \\
\text { blema Tipo } \\
\text { (15 min) }\end{array}$ & $\begin{array}{l}\text { El profesor resuelve } \\
\text { en la pizarra un pro- } \\
\text { blema tipo: desa- } \\
\text { rrollo de contenidos } \\
\text { procedimentales }\end{array}$ & $\begin{array}{l}\text { - Transferencia de co- } \\
\text { nocimiento en el } \\
\text { procedimiento de reso- } \\
\text { lución de problemas }\end{array}$ \\
\hline 7 & $\begin{array}{l}\text { Resolución Pro- } \\
\text { blemas por los } \\
\text { alumnos } \\
\text { (25 min) }\end{array}$ & $\begin{array}{l}\text { El profesor propone } \\
\text { problemas a los es- } \\
\text { tudiantes que deben } \\
\text { resolver por ellos mis- } \\
\text { mos buscando infor- } \\
\text { mación y trabajando } \\
\text { en equipo }\end{array}$ & $\begin{array}{l}\text { - Conocer el grado de } \\
\text { comprensión de las } \\
\text { materias impartidas } \\
\text { - Aflorar dudas latentes } \\
\text { Participación colectiva }\end{array}$ \\
\hline 8 & $\begin{array}{l}\text { Autoevaluación } \\
\text { (5 min) }\end{array}$ & $\begin{array}{l}\text { El profesor propone } \\
\text { un problema volun- } \\
\text { tario para trabajar en } \\
\text { casa }\end{array}$ & $\begin{array}{l}\text { - Que los alumnos tra- } \\
\text { bajen a diario y sean } \\
\text { conscientes de sus } \\
\text { avances }\end{array}$ \\
\hline 10 & Fudas (5min) & $\begin{array}{l}\text { El profesor resuelve } \\
\text { dudas que queden sin } \\
\text { resolver }\end{array}$ & $\begin{array}{l}\text { - Resolver dudas de los } \\
\text { alumnos }\end{array}$ \\
\hline & $\begin{array}{l}\text { El profesor ayuda a } \\
\text { que los propios alum- } \\
\text { nos identifiquen las } \\
\text { ideas principales de la } \\
\text { sesión }\end{array}$ & $\begin{array}{l}\text { - Cierre de la sesión } \\
\text { Conclusiones }\end{array}$ \\
\hline
\end{tabular}

Los principales puntos de interés en el desarrollo de las actividades, para cada una de las tres fases que componen el Modelo Metodológico, fueron:

\section{FASE 1: Inicio}

- Los alumnos recuerdan y participan activamente en el repaso de los contenidos de la sección anterior.

- En el planteamiento de la "pregunta inicial" tractora de la motivación y curiosidad de los estudiantes, estos responden con entusiasmo tratando de indagar soluciones preliminares. Se genera un ambiente colaborativo en el aula.

- Se reparte el cuestionario inicial y aunque los alumnos al principio permanecen reacios a cumplimentarlos porque piensan que puede afectar a su 
calificación; aclarado este aspecto y que el cuestionario es solo para cuantificar el grado de aprendizaje de esta sección y que el resultado no es puntuable para la asignatura, el ambiente es más participativo.

Es una evidencia clara que comenzar la clase situando al alumno con un problema que no saben responder a priori es positivo para captar su atención durante la sesión. El alumno es consciente de que le falta conocimiento para abordar la resolución del problema y se siente entonces partícipe del proceso de aprendizaje.

La realización del cuestionario inicial es positivo para el profesor: le permite conocer conocimientos previos y obstáculos presentes en los alumnos, y así poder rediseñar las sesiones de la sección para nivelar el aprendizaje al final de la misma. Lo que no se mide no se mejora. Es una buena práctica este planteamiento inicial. Por otro lado, el inconveniente que se observa es que el Proyecto Docente de la asignatura está muy ajustado al tiempo en aula e invertir tiempo en estas actividades no planificadas inicialmente obliga a una revisión de contenidos, que en el caso adicional de ser una asignatura que se imparte en cuatro grupos con otros profesores, requiere de coordinación y consenso.

\section{FASE 2: Desarrollo}

- Se han impartido conocimientos teóricos propios de la sección que estaba planificada. El método ha sido generando preguntas para que los alumnos fueran construyendo su propio conocimiento. Se observa además como beneficio no esperado, que al crear un ambiente más participativo y abandonar el profesor su papel de protagonista expositivo, se facilita a que alumnos reconozcan dudas que tienen en la comprensión de la sección; en otras condiciones, probablemente estos alumnos no hubieran preguntado 
sus dudas y se hubiera generado un obstáculo innecesario en su aprendizaje.

- Para facilitar la participación y que los alumnos sean los protagonistas de la clase, se hace uso de herramientas TIC, concretamente Socrative. En este punto hay que mencionar que esta actividad ya se había planteado en otra sección anterior con escasa participación y entusiasmo por los alumnos. En este caso ocurre lo contrario: los alumnos se animan en la resolución de las preguntas que se han planteado.
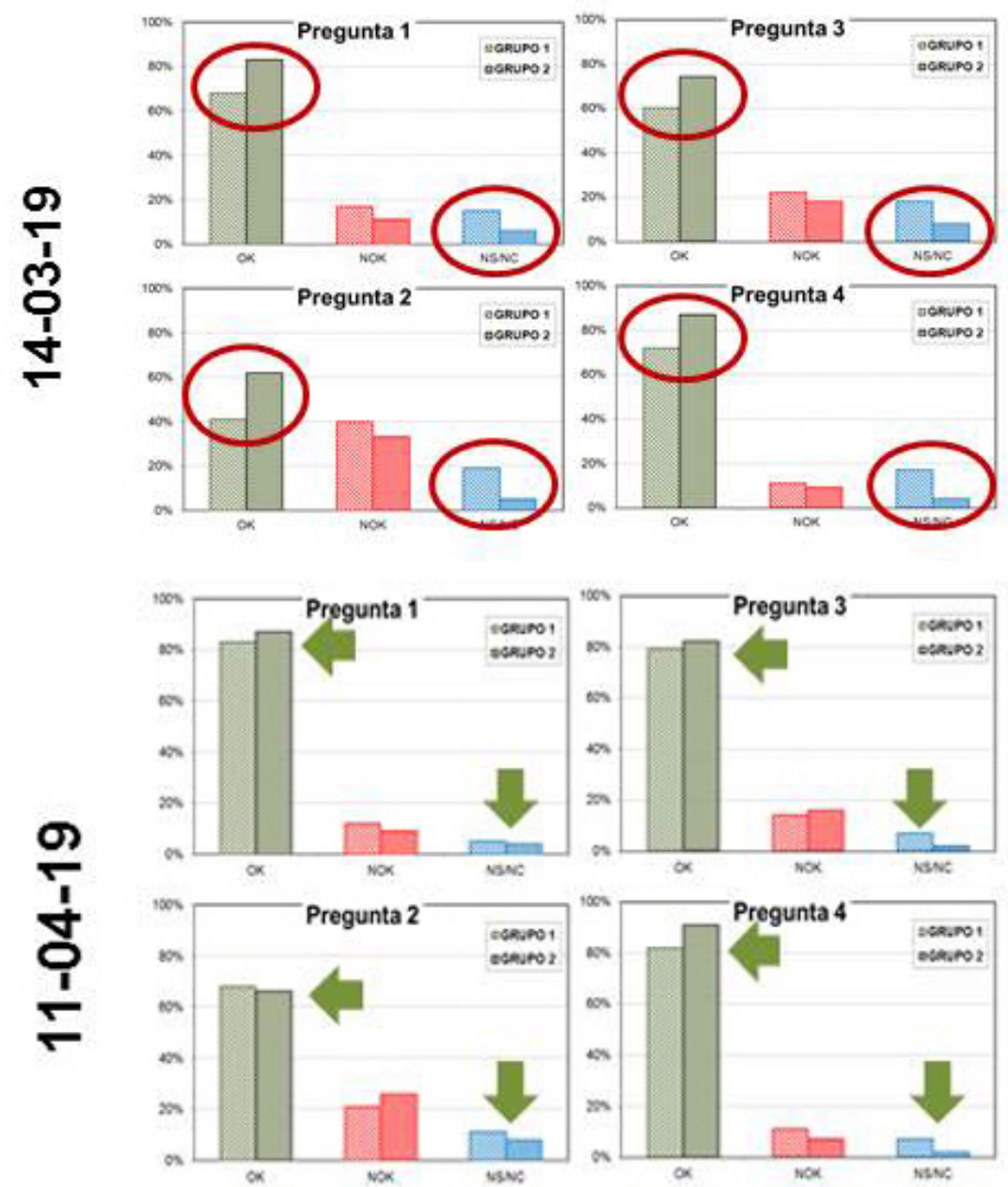

Figura 5. Resultados de la actividad participativa de Socrative, comparando resultados de diferentes grupos y diferentes sesiones

Jornadas de Formación e Innovación Docente del Profesorado | № 2 (2019) Esta obra se distribuye con la licencia Creative Commons Reconocimiento-NoComercial-SinObraDerivada 
Cuando se analizan los resultados obtenidos en el ciclo de Mejora comparados con otros resultados previos (Figura 5), se observa que en general el nivel de participación de la actividad es positivo y que la mayor parte de los alumnos responde satisfactoriamente a todas las preguntas planteadas; hace entender al profesor que los contenidos de la sesión se han comprendido. En relación a los resultados previos, en esta ocasión no hay diferencias significativas entre los dos grupos donde se ha puesto en práctica, ni en participación ni en grado de aprendizaje. Es decir, podemos concluir que otra ventaja positiva del Ciclo de Mejora es que se ha uniformizado el aprendizaje adquirido por los estudiantes en diferentes grupos de la asignatura.

- La resolución de los casos prácticos comienza con un ejercicio tipo por parte del profesor en la pizarra; sirve para indicar el procedimiento de resolución y asentar aspectos teóricos anteriores. A continuación, el resto de ejercicios que se realizan en clase no los hace el profesor sino los alumnos, trabajando en modo colaborativo. Cuando se finalizan son ellos mismos quienes exponen en común los resultados y en caso de discrepancias, debaten y argumentan sus soluciones. Esto fortalece trabajar además de los contenidos de la asignatura, aquellas competencias que los alumnos deben desarrollar para su futuro profesional: trabajo en equipo, exposición de resultados, argumentación, empatía, etc.

Cuando algún alumno tenía dudas y preguntaba al profesor, la duda era derivada a sus propios compañeros: se observa que además el lenguaje que usan entre ellos facilita la comunicación del mensaje de manera más fluida.

El tiempo que ellos dedican a resolver los problemas por sí mismos, el profesor pasea entre ellos observando y tutorizando, pero en un papel secundario. Esta cercanía que se genera entre el profesor y los 
estudiantes también se aprovecha para conocer con más detalle los diferentes grados de conocimiento que los estudiantes tienen de la asignatura, y así poder dedicar un tiempo más personalizado a aquellos alumnos que tienen dudas.

\section{FASE 3: Cierre}

- Se propone un ejercicio de autoevaluación que los estudiantes tienen que trabajar de manera voluntaria, fuera del tiempo de aula. Esto permite identificar los conocimientos falsamente comprendidos y poner por tanto solución a ello antes del examen. Al profesor además le permite saber el grado de avance en el aprendizaje de los estudiantes.

- Una vez enunciado el problema a resolver, el profesor entrega los ejercicios corregidos que se propusieron la semana anterior. Esto facilita también al alumno conocer el método de corrección que el profesor va a imponer en el examen, con tiempo suficiente para prever problemas. El ejercicio se entrega corregido y con anotaciones, pero no evaluado. Es el propio alumno quien revisa su trabajo, las anotaciones del profesor y se autoevalúa.

- Esta es una actividad voluntaria, que se anuncia que no tiene peso en la calificación final de la asignatura, pero que ofrece ventajas para el aprendizaje. Se ha observado cómo semana a semana de forma voluntaria, el número de alumnos que participan de esta actividad ha ido en aumento.

- Se abre un tiempo para plantear dudas y se resuelven; en esta ocasión se observa que el número de dudas latentes a final de la clase es menor que en otras ocasiones, probablemente porque la mayoría de ellas han quedado resueltas durante las interacciones alumno - profesor en el desarrollo de la clase. 
- Resumen de las ideas principales de la sesión y visualización de contenidos con el Mapa de Contenidos que se comparte con los estudiantes.

- El Ciclo de Mejora se aplicó durante 4 clases (Jueves y Viernes de dos semanas, cada clase de $110 \mathrm{mi}$ nutos), en dos grupos en paralelo. En la última de las sesiones en esta fase se planteó a los alumnos el cuestionario final (Figura 4). Los resultados serán posteriormente analizados para generar las escaleras de aprendizaje.

\section{Evaluación del aprendizaje}

Se analizan las respuestas a los cuestionarios inicial y final, con el fin de evaluar el grado de aprendizaje de los estudiantes (Porlán, 2017). El número de alumnos que han participado en los cuestionarios inicial y final no ha sido el mismo, pero se analizan solo aquellos cuestionarios que han sido coincidentes en su cumplimentación por el mismo estudiante al inicio y fin de la sesión. El total de cuestionarios analizados así es de 171, lo que supone aproximadamente un $35 \%$ de los alumnos matriculados en la asignatura.

El criterio de evaluación consistió en identificar cuatro niveles de aprendizaje:

A: respuesta correcta

B: respuesta parcialmente correcta

C: respuesta incorrecta aunque hay algún conocimiento latente en el estudiante

D: no se responde o la respuesta es muy incorrecta A partir del análisis de las respuestas para cada pregunta, se construyen las escaleras de aprendizaje ( $\mathrm{Fi}$ guras 6a, 6B y 6C).

Jornadas de Formación e Innovación Docente del Profesorado | № 2 (2019) Esta obra se distribuye con la licencia Creative Commons Reconocimiento-NoComercial-SinObraDerivada 
స 1.A. Describir brevemente la finalidad de un ciclo termodinámico

․ㅡ. Tipos de ciclos y principal diferencia entre ellos

1.C. Dibuje diagrama termodinamico de cuatro ciclos alternativos

프 1.A. Cómo maximizar eficiencia de un ciclo termodinámico

ㄴ 1.B. Dibuje las interacciones energéticas en un ciclo de flujo y uno alternativo

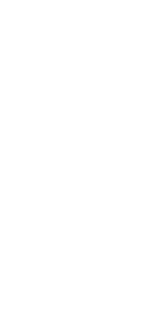

BLOQUE 1 - CICLOS TERMODINAMICOS

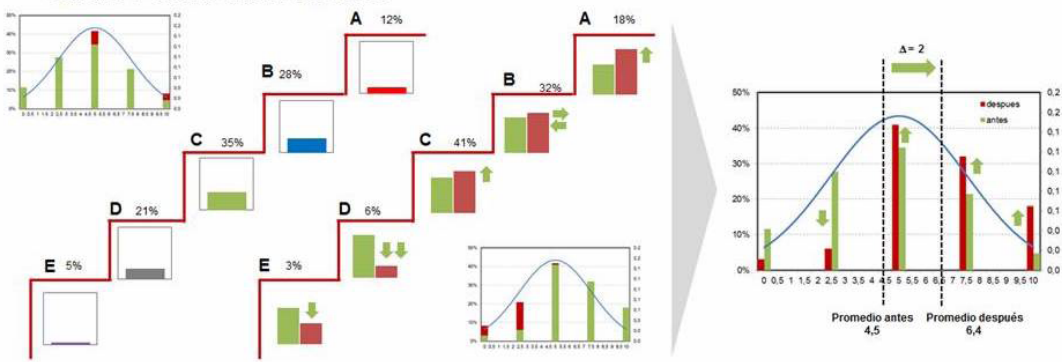

$\frac{\overline{0}}{0}$ 2.A. Finalidad del Primer Principio de la Termodinamica

2 즐 2.A. Limitaciones del Primer Principio de la Termodinamica

ㄴ. 2.B. Limitaciones del Segundo Principio de la Termodinamica

BLOQUE 2 - PRINCIPIOS DE LA TERMODINAMICA
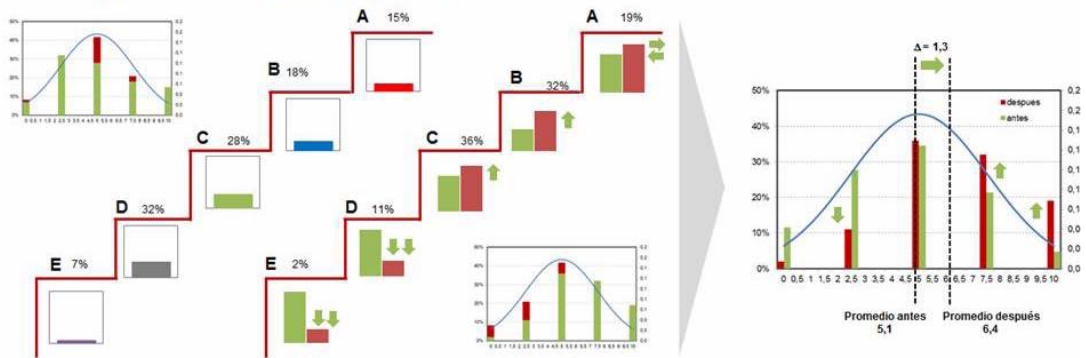

ত্ত 3.A. Exergía: ¿qué representa y por qué es de interés el concepto?

3.B. Expresión de la exergía para un sistema abierto con dos entradas, una salida, !

3 en presencia de un reservorio de calor y ambiente, en el límite termodinámico

$\overline{\mathbb{C}}$ 3.A. Exergía: ¿valor en un sistema en condiciones ambiente?

드 3. B. Expresión de la exergía para un sistema abierto con una entrada, dos salidas, en presencia de un reservorio de trabajo y ambiente, en el límite termodinámico

BLOQUE 3 - EXERGIA
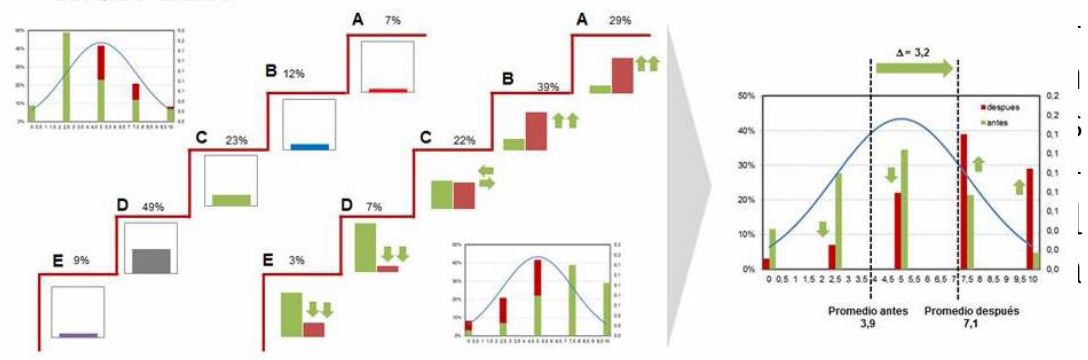

Figura 6. Generación de escaleras de aprendizaje para cada pregunta del cuestionario

Jornadas de Formación e Innovación Docente del Profesorado I № 2 (2019) Esta obra se distribuye con la licencia Creative Commons Reconocimiento-NoComercial-SinObraDerivada Internacional (CC BY-NC-ND 4.0.) 
Las escaleras de aprendizaje generadas también son interesantes no sólo para medir el progreso de conocimiento alcanzado en los estudiantes, sino la identificación de barreras u obstáculos que han aparecido (Figura 7). Estos deberían servir para:

a) Precaución en el examen si se preguntan estos conceptos porque no parecen claros en una mayoría significativa.

b) Revisar contenidos o modelo metodológico en futuras sesiones por parte del profesor.

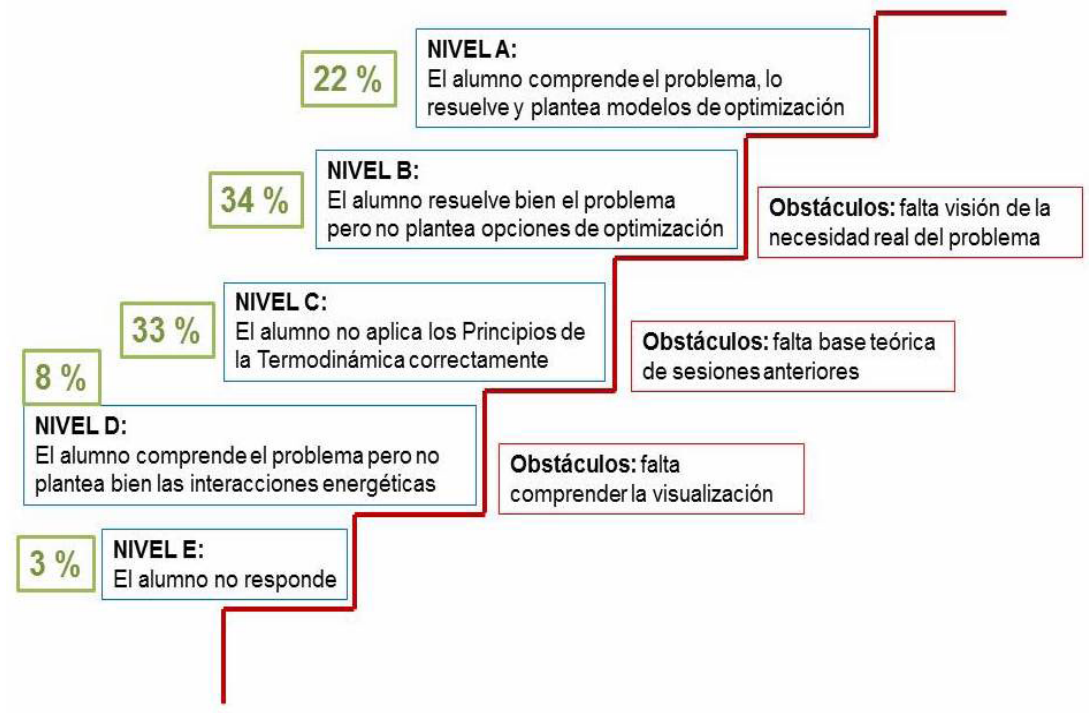

Figura 7. Identificación de obstáculos (derecha en rojo) y del grado de aprendizaje de los contenidos (izquierda en azul)

Por último, se presenta una interpretación de las escaleras de aprendizaje según el modelo de Corrochano (Porlán, 2017). En esta interpretación se analizan los obstáculos en el aprendizaje de los estudiantes según las escaleras de aprendizaje, identificando incluso subniveles en ellas.

Jornadas de Formación e Innovación Docente del Profesorado | № 2 (2019) Esta obra se distribuye con la licencia Creative Commons Reconocimiento-NoComercial-SinObraDerivada Internacional (CC BY-NC-ND 4.0.) 
Tabla 2. Escalera de aprendizaje según el modelo de Corrochano

\begin{tabular}{|c|c|c|}
\hline \multicolumn{2}{|c|}{ MODELOS DETECTADOS } & \% ESTUDIANTES \\
\hline \multirow{4}{*}{$\begin{array}{l}\text { Grupo A: los alumnos } \\
\text { resuelven un ciclo ter- } \\
\text { modinámico y optimizan } \\
\text { su rendimiento }\end{array}$} & $\begin{array}{l}\text { A.1. Resolución basada en } \\
\text { el balance de exergía }\end{array}$ & $1 \%$ \\
\hline & $\begin{array}{l}\text { A.2. Discusión del límite } \\
\text { termodinámico }\end{array}$ & $11 \%$ \\
\hline & $\begin{array}{l}\text { A.3. Comparativa ren- } \\
\text { dimiento energético y } \\
\text { exergético }\end{array}$ & $6 \%$ \\
\hline & $\begin{array}{l}\text { A.4. Discusión de pro- } \\
\text { puestos de optimización }\end{array}$ & $3 \%$ \\
\hline \multicolumn{2}{|c|}{$\begin{array}{l}\text { Grupo B: los alumnos no recuerdan el concepto de } \\
\text { exergía y no plantean una optimización o discusión } \\
\text { del proceso analizado }\end{array}$} & $34 \%$ \\
\hline \multicolumn{2}{|c|}{$\begin{array}{l}\text { Grupo C: los alumnos no recuerdan correctamente } \\
\text { los Principios de la Termodinámica y no tienen base } \\
\text { teórica para resolver el problema que se les plantea }\end{array}$} & $33 \%$ \\
\hline \multicolumn{2}{|c|}{$\begin{array}{l}\text { Grupo D: los alumnos realizan un dibujo de las inte- } \\
\text { racciones en el diagrama termodinámico (visualiza- } \\
\text { ción del problema) incorrecto }\end{array}$} & $8 \%$ \\
\hline \multicolumn{2}{|c|}{ Grupo E: los alumnos no responden a la pregunta } & $3 \%$ \\
\hline
\end{tabular}

\section{Evaluación del ciclo de mejora puesto en práctica}

El balance general de la práctica del ciclo de mejora en el aula que se ha diseñado en la asignatura de Termodinámica, ha sido positivo; tanto a nivel mío personal como por los comentarios recibidos de los alumnos. Se ha profundizado en el aprendizaje de los contenidos de la asignatura mediante herramientas o actividades alternativas a la "clase magistral" tradicional, y las actividades de evaluación (Socrative, cuestionario, problemas de autoevaluación y problemas en clase) indican que los conocimientos han quedado bien asentados en los alumnos. 
Además el modelo metodológico puesto en práctica ha permitido fomentar competencias en los alumnos que serán de gran utilidad en su futuro desarrollar: pensamiento crítico, trabajo en equipo, exposición de resultados, argumentación, debate, empatía y respecto, etc. Las herramientas empleadas también son en parte transferidas a los estudiantes para que ellos mismos las empleen en sus horas de estudio personales, como por ejemplo la elaboración de los Mapas de Contenidos.

Destacaría por encima de todo el hecho de comenzar la sesión planteando un interrogante el alumno, con trascendencia técnica y social, que les implique en el porqué del diseño de los contenidos de la asignatura. Una pregunta que les sirva de tracción al interés por aprender. Una pregunta que les una al profesor durante la clase e invierta los papeles protagonistas siendo ellos el centro del desarrollo del conocimiento y no unos meros espectadores.

Pero sin duda nada de esto sería posible al interés del alumnado presente en las sesiones. Es claro que el compromiso por innovar en la metodología docente tiene que ser y ha sido en este caso, de ambas partes. Sería un acierto implementar este modelo metodológico en próximos cursos académicos, si bien es cierto que sería necesaria una revisión de los contenidos en el Proyecto Docente de la asignatura en consenso con el resto de profesores que la imparten. El tiempo que requiere este modelo didáctico alternativo es claramente superior a la clase magistral.

El hecho además de poner en práctica este modelo metodológico en un aula con número de estudiantes elevados puede también significar un obstáculo, pues limita la participación de todos los estudiantes y un seguimiento más exhaustivo del profesor hacia ellos. Preguntado a los estudiantes por su grado de satisfacción, resaltar que aunque es un porcentaje pequeño pero significativo, hay una pequeña población de ellos que prefieren el modelo 
tradicional de clase magistral porque en esta alternativa docente manifiestan sentirse más desorientados en la asignatura al ser ellos quienes deben trabajar en construir los contenidos. Creo que tal vez una aproximación apropiada sería alternar ambos modelos metodológicos con menor peso en la clase magistral, e incidir en niveles inferiores académicos para que los estudiantes universitarios estén más habituados a aprender en base a problemas (ABP).

Como línea de trabajo abierta, sería interesante plantear los cuestionarios y elaboración de escaleras de aprendizaje en varias etapas del cuatrimestre, y finalmente compararlas con las calificaciones del examen final. Esta comparación pondría de manifiesto si (1) se corresponde el aprendizaje en evaluación continua con el examen final, y (2) si en tal caso podría bajarse el peso de la calificación del examen en aras de otras actividades a lo largo del cuatrimestre.

\section{Conclusiones}

- La principal y general conclusión es que la experiencia de implementar el Modelo Metodológico descrito en el Ciclo de Mejora ha sido positiva. El cambio implementado para que el centro de atención en el aula sea el alumno trae como consecuencias positivas: (a) el alumno es consciente de su aprendizaje y por tanto este proceso es más eficiente; (b) el alumno desarrolla mayor autonomía en su aprendizaje; (c) se facilita una mayor comunicación entre el profesor y alumno que permite detectar obstáculos con mayor rapidez.

- El uso de herramientas como el Mapa de Contenido es beneficiosa para revisar contenidos, identificar aquellos que verdaderamente son prioritarios, establecer relaciones entre ellos, etc. 
- Excelente práctica iniciar las clases con una "pregunta inicial" que sea tractora del interés, curiosidad y motivación del alumno. Esta pregunta además contextualiza al estudiante en el Proyecto Docente.

- Elaborar cuestionarios iniciales y finales en diferentes etapas del cuatrimestre es otra práctica recomendable y complementaria con los problemas de autoevaluación. Detecta obstáculos y genera información del nivel de evolución de aprendizaje por parte de los estudiantes.

- Las conclusiones positivas de la puesta en práctica del Ciclo de Mejora presente, no excluyen de forma completa la docencia basada en "clases magistrales". Para los alumnos, en determinados conceptos, es beneficioso también disponer del método de enseñanza tradicional. Se recomienda por tanto un modelo mixto donde ambos modelos de aprendizaje son compatibles.

Jornadas de Formación e Innovación Docente del Profesorado | № 2 (2019) Esta obra se distribuye con la licencia Creative Commons 


\section{Referencias bibliográficas}

Beard, R. y Hartley, J. (1984). Teaching and learning in higher education. Londres: Harper Row.

Finkel, D. (2008) Dar clase con la boca cerrada. Valencia: Universitat de Valencia.

Hutchens, D. (2009) De la caverna a la consciencia. Madrid: Griker Orgemer.

Porlán R. (Coord.) (2017). Enseñanza universitaria. Cómo mejorarla. Madrid: Morata. 